\title{
Measuring financial risk and portfolio reversion with time changed tempered stable Lévy processes
}

\begin{abstract}
Given that underlying assets in financial markets exhibit stylized facts such as leptokurtosis, asymmetry, clustering properties and heteroskedasticity effect, this paper applies the stochastic volatility models driven by tempered stable Lévy processes to construct time changed tempered stable Lévy processes (TSSV) for financial risk measurement and portfolio reversion. The TSSV model framework permits infinite activity jump behaviors of returns dynamics and time varying volatility consistently observed in financial markets by introducing time changing volatility into tempered stable processes which specially refer to normal tempered stable (NTS) distribution as well as classical tempered stable (CTS) distribution, capturing leptokurtosis, fat tailedness and asymmetry features of returns in addition to volatility clustering effect in stochastic volatility. Through employing the analytical characteristic function and fast Fourier transform (FFT) technique, the closed form formulas for probability density function (PDF) of returns, value at risk (VaR) and conditional value at risk (CVaR) can be derived. Finally, in order to forecast extreme events and volatile market, we perform empirical researches on Hangseng index to measure risks and construct portfolio based on risk adjusted reward risk stock selection criteria employing TSSV models, with the stochastic volatility normal tempered stable (NTSSV) model producing superior performances relative to others.
\end{abstract}

Keywords: stochastic volatility; tempered stable Lévy process; financial risk measurement; portfolio reversion

\section{Introduction}

Financial risk measurement and portfolio reversion closely rely on the modeling of underlying asset dynamics (Kim et al., 2011; Goode et al., 2015). Abundant evidence has contradicted the normal distribution assumption in returns distribution which exhibits leptokurtosis, asymmetry and volatility clustering phenomena, leading to stochastic jumps in stock prices. It has been demonstrated by Ait-Sahalia et al. (2012) and Klingler et al. (2013) that stochastic volatility and jumps are inherent components of stock price dynamics which play important roles in accounting for stylized facts in financial markets. Therefore, when constructing models to describe asset dynamics, it is necessary to incorporate both stochastic volatility and leptokurtosis components. Vast efforts have been made to relax these restrictive assumptions. The considerably observed stylized facts comprising abnormality and heteroskedasticity induce extensive alternative models accommodating stochastic volatility in returns variance and infinite jumps behaviors in price processes (Forsberg, 2002; Su, 2011).

One alternative way to capture the high peak and fat tail property in financial markets is to employ the infinite activity tempered stable (TS) processes that belong to Lévy processes and have been validated superior to the $\alpha$ stable Paretian distribution, which has been demonstrated insufficient to explain the dynamics of stock processes flexibly. Since the $\alpha$ stable Paretian distribution has infinite moments, the introduction of tempered stable distribution presented in Rosinski (2007) enhances it effectively in accounting for heavy tails and asymmetry in empirical data within converged moments. Just as what Carr and $\mathrm{Wu}$ (2004) have addressed that infinite activity Lévy processes can capture both large jumps and small jumps in stock price dynamics that are consistently observed in financial markets. Compared with other infinite activity Lévy processes such as the Variance Gamma process (VG) presented by Madan and Carr (1998), Normal Inverse Gaussian process (NIG) presented by Barndorff Nielsen (1997), the infinite activity tempered stable Lévy models are attractive since they can capture jump patterns exhibited by stock returns more effectively when combined with stochastic volatility components (Gong et al. 2016). The Lévy process can be infinite activity processes that generate infinite jumps within finite interval or it can be finite activity processes that generate finite jumps. We especially focus on the case where the Lévy jumps are infinite to capture both large and small jumps in stock returns.

The tempered stable processes consist of classical tempered stable distribution (CTS) process, normal tempered stable distribution (NTS) process and rapidly decreasing tempered stable distribution process in Kim et al. (2010) and so on. Noticeably, the CGMY distribution proposed by Carr et al. (2002) is one of the special cases of infinite activity tempered stable distributions which is extensively studied by Kuchler et al. (2013) and Zaevski et al. (2014). The TS 
distributions are gained by making $\alpha$ stable law multiply their corresponding Lévy measures generated from moment generating functions. This type of distribution is equipped with more proper ability in capturing asymmetry, higher kurtosis and fatter tails than $\alpha$ stable distribution, whose tail distributions are too thick to capture empirical tail behaviors in markets. Moreover, its infinite activity characteristic can better describe the infinite jumps phenomenon exists in the underlying process (Gajda et al., 2013; Ruan, 2016). In addition, the attainability of closed form characteristic functions of Lévy processes makes it possible for deriving density functions of these distributions employing fast Fourier transform method as well as calculating value at risk and conditional value at risk.

Another alternative approach to tackle heteroskedasticity and volatility clustering properties in returns variance is the application of stochastic volatility models. Compared to the single factor volatility model like GARCH model, the continuous time stochastic volatility model incorporates random volatility in both return process and volatility process, which is better for financial modeling. Regarding the time varying volatility in returns variance, Ouadghiri et al. (2016) performed research on stochastic volatility models with jumps components. Although plentiful research has been performed on jump-diffusion stochastic volatility models (Todorov, 2011; Andreas et al., 2013, Kim et al., 2008), they feature a counter-factual assumption that jumps rarely occur. It is reasonable to substitute finite jump components into infinite Lévy jumps. Huang and $\mathrm{Wu}$ (2004) demonstrated that process models incorporating both infinite jumps and stochastic diffusion components are appropriate in option markets. The same conclusion can be inferred from Carr and $\mathrm{Wu}$ (2004), who expressed that introducing stochastic volatility into Lévy processes permitted reproducing excessive skews, kurtosis and persistent heteroskedasticity in underlying asset process. In order to capture both these properties, Schoutens et al. (2003) proposed the Lévy driven stochastic volatility models framework that employ time changed Lévy processes out of a Cox-Ingersoll-Ross (CIR) square root stochastic clock and it included processes such as VGCIR process and NIGCIR process and so on. By increasing or decreasing the uncertainty in economy through speeded or slowed elapsed time rate. Zaevski et al. (2014) turned attention to the stochastic volatility model with classical tempered stable Lévy jumps in order to describe the leptokurtosis, asymmetry property in returns and varying volatility. We enhance the prevailing model from time changed classical tempered stable distribution (CTSSV) to time changed normal tempered stable distribution (NTSSV) to reconstruct the time changed tempered stable model framework.

The empirical evidence of skewed and leptokurtic nature in asset returns have important implications in risk management. The failures of risk management during crisis call for more suitable financial models for describing returns distribution (Wilhelmsson, 2009; Huang, 2015). Our proposed TSSV modeling framework together with risk measure of conditional value at risk will be illustrated more effective in risk assessment. The CVaR measure deals with the limitations of VaR measure (Han, 2014), it has been illustrated robust with respect to tail index in Stoyanov et al. (2013), and require taking into consideration appropriately the clustering of volatility. Stock selection criteria play significant roles in portfolio formulation (Georgiev, 2015). The usual criterion is the Sharpe ratio that is valid when asset returns are Gaussian distributed. The overwhelming evidence on asymmetries and fat tails leads to alternative stock selection measure. Furthermore, we analyze portfolio strategies on the basis of reward risk stock selection criteria utilizing CVaR as risk measures of reward.

The improvements contribution of our work over the prevailing is that we subordinate normal tempered stable processes to time varying stochastic volatility processes to construct time changed tempered stable models framework, capturing the distributional behavior of empirical data. By exploiting Fourier transform technique for probability calculation, the closed form solutions for $\mathrm{PDF}$, cumulative density function $(\mathrm{CDF})$ and $\mathrm{VaR}$ as well as $\mathrm{CVaR}$ are derived. Then, we evaluate the risk profile of Hangseng index to capture stylized facts and apply alternative risk adjusted stock selection criteria for portfolio performance investigation.

The remainder of the paper is organized as follows. Time changed tempered stable Lévy processes framework is reconstructed by subordinating infinite activity tempered stable Lévy processes to the integrated square root CIR process in Section 2. In Section 3, by utilizing the Fourier transform of the corresponding characteristic functions of time changed tempered stable Lévy processes, analytical expressions of their probability density functions are derived, thus the $\mathrm{VaR}_{\delta}$ and $\mathrm{CVaR}_{\delta}$ can be calculated. The alternative risk-adjusted criteria including $\mathrm{STAR}_{(1-\delta) 100 \%}$ 
ratio and $\mathbf{R}_{(\alpha, \beta)}$ ratio for stock ranking as well as their reasons are listed in Section 4. In Section 5, the backtesting consisting of the Christoffersen likelihood ratio test and the Berkowitz likelihood ratio test for different models are performed to evaluate the accuracy of forecasting, and then we investigate the portfolio strategy of index constituent stocks with different models under different ratios, and give the distributional analysis of portfolio under TSSV. In Section 6, we summarize our principal findings.

\section{Time changed tempered stable Lévy processes}

\subsection{Infinite activity tempered stable Lévy processes}

Let $\varphi$ represent the characteristic function of infinite activity tempered stable Lévy processes, $X_{t}$ denote stock returns. We define the Lévy process $X(t)$ in filtration space $\left(\Omega, F \_t, P\right)$ an infinite divisible distribution which has independent and stationary increments as well as $n$th power of characteristic functions $\varphi(u)$. The infinite activity Lévy distribution has a triplet of characteristics $(\mu, \sigma, v)$ (Sato, 1999), where it denotes a linear deterministic component, a Brownian motion component and a pure jump part, respectively. Given that the distribution function forms for Lévy processes are generally complicated, we employ characteristic function expression $\varphi(u)=E\left[e^{i u X(t)}\right]$, or characteristic exponent $\psi(u)=\log \varphi(u)$ expression for convenience. The characteristic function $\varphi(u)$ meets the following equation according to Lévy-Khintchine formula:

$$
E[\exp (i u X)]=\exp \left(-i u \mu+\frac{\sigma^{2} u^{2}}{2}-\int_{-\infty}^{+\infty}\left(e^{i u x}-1-i u x_{|x| \leq 1}\right) v(d x)\right)
$$

where $\mu$ represents the drift that controls the location of distribution, $v$ refers to the Lévy measure which suggests the arrival rate of jumps.

It is known that Lévy measure obtained from the FFT of characteristic functions plays significant role in reflecting jump behaviors and jumps structure of random distributions in addition to reflecting the jump intensity differences for different processes. The Lévy measure is associated with probability density distribution of the corresponding process, with its distributional width related to the peak of density function, and its distributional height associated with the tail of density function. The corresponding Lévy measure of VG distribution, CTS distribution and NTS distribution are as follows:

$$
\begin{gathered}
v_{V G}(d x)=\left\{\begin{array}{l}
\mathrm{C}_{1} \exp (\mathrm{G} x) /|x| 1_{(x<0)} \\
\mathrm{C}_{1} \exp (-\mathrm{M} x) / x 1_{(x>0)}
\end{array}\right. \\
v_{C T S}(d x)=\frac{\mathrm{C}_{2} e^{-\lambda_{+} x}}{x^{1+\alpha}} 1_{x>0}+\frac{\mathrm{C}_{2} e^{-\lambda-|x|}}{|x|^{1+\alpha}} 1_{x<0} \\
v_{N T S}(d x)=\sqrt{\frac{2}{\pi}}\left(\frac{v}{1-\theta}\right)^{\theta-1} \frac{e^{\frac{\mu x}{\sigma^{2}}}|x|^{-\theta-\frac{1}{2}}}{\sigma \Gamma(1-\theta)}\left(\mu^{2}-\frac{2(\theta-1) \sigma^{2}}{v}\right)^{\frac{\theta}{2}+\frac{1}{4}} K_{\theta+\frac{1}{2}}\left(\sqrt{\frac{x^{2}}{\sigma^{2}}\left(\frac{\mu^{2}}{\sigma^{2}}-\frac{2(\theta-1)}{v}\right)}\right)
\end{gathered}
$$

where $K$ denotes the modified Bessel function of the third kind. $v_{C T S}(\mathrm{R})=v_{N T S}(\mathrm{R})=\infty$, indicating that the CTS and NTS processes are pure jumps processes and possess infinite activity property.

We suppose the returns process to follow tempered stable distribution and particularly pay attention to three kinds of tempered stable distributions: the Variance Gamma distribution, the classical tempered stable distribution and the normal tempered stable distribution. The characteristic functions of VG distribution, CTS distribution and NTS distribution are calculated as:

$$
\begin{gathered}
\varphi_{V G_{X_{t}}}(u)=\mathrm{C}_{1} \log \left[\mathrm{GM} /\left(\mathrm{GM}+(\mathrm{M}-\mathrm{G}) i u+u^{2}\right)\right] \\
\varphi_{C T S_{X_{t}}}(u)=\exp \left(t \mathrm{C}_{2} \Gamma(-\alpha)\left(\lambda_{-}-i u\right)^{\alpha}-\lambda_{-}^{\alpha}+\left(\lambda_{+}+i u\right)^{\alpha}-\lambda_{+}^{\alpha}\right) \\
\varphi_{N T S_{X_{t}}}(u)=\exp \left(\frac{(1-\theta) t}{v \theta}\left(1-\left(1-\frac{\left(i u \mu-\frac{\sigma^{2} u^{2}}{2}\right) v}{1-\theta}\right)^{\theta}\right)\right)
\end{gathered}
$$

where $\Gamma$ is the gamma function, $\mathrm{C}_{1}, \mathrm{G}, \mathrm{M}$ denote parameters in Variance Gamma distribution, $\mathrm{C}_{2}$, $\lambda_{-}, \lambda_{+}, \alpha$ denote parameters in classical tempered stable distribution model respectively, $\alpha \in(0,2)$ and $\alpha \neq 0, \lambda_{-}, \lambda_{+}, \alpha>0$. And $\mu, v, \sigma, \theta$ denote parameters in normal tempered stable distribution model 
respectively, where $\mu \in \mathrm{R}, \theta \in(0,1), v, \sigma>0$. Using above characteristic functions, the cumulant $c_{n}$ of tempered stable processes can be calculated through

$$
c_{n}=\frac{1}{i^{n}} \frac{d^{n}}{d u^{n}} \log E\left[e^{i u X}\right]
$$

In VG model, parameter $\mathrm{C}_{1}$ governs the total activity rate of the process, while parameters $\mathrm{G}$ and $\mathrm{M}$ influence the arrival rates of jumps in opposite upward and downward jumps directions. The scale parameter $\mathrm{C}_{2}$ in CTS model associates with kurtosis of the density function, with smaller $C_{2}$ resulting to higher peak. While parameters $\lambda_{+}$and $\lambda_{-}$in classical tempered stable distribution grasp the tails decay rate in positive and negative sides, respectively, with parameters $\lambda_{-}, \lambda_{+}, \alpha$ related to the tails weights. If $\lambda_{+}$disagrees with $\lambda_{-}$, it is asymmetric in jumps structure. When $\lambda_{+}>\lambda_{-}\left(\lambda_{-}>\lambda_{+}\right)$, the returns distribution is left (right) skewed, otherwise, it exhibits symmetry in returns. In addition, parameter $\mu$ in normal tempered stable distribution determines the skewness of density function, $v$ controls the excessive kurtosis exceeding normal distribution, $\theta$ affects the shape of returns distribution. When specifying $\theta=1 / 2$, it gives rise to the case of Normal Inverse Gaussian model, while approximating to zero, it leads to the case of Variance Gamma model. What can be concluded from above is that higher kurtosis in time series corresponds to higher value of $\theta$.

\subsection{Time changed tempered stable Lévy processes}

The stochastic variation in volatility can be featured by instantaneous changes in time. The model utilized to induce stochastic volatility is the integrated square root CIR process, which describes the volatility dynamic $y_{t}$ utilizing $\operatorname{SDE} d y_{t}=\kappa\left(\eta-y_{t}\right) d t+\lambda \sqrt{y_{t}} d W_{t}$, with the corresponding parameters $\kappa$ denoting the speed of mean reversion, $\eta$ represents the long term variance, $\lambda$ stands for the volatility of volatility. The CIR mean reverting process acts as measurement of time change rate through speeding up or slowing down the elapsed time according to the economic events so as to increase or decrease the activity intensity, thus capturing the volatility clustering and heteroskedasticity effect.

The reason why we choose the mean reverting CIR process for measuring the time change rate in building model for volatility clustering is that the economic time $Y_{t}$ passed in $t$ units time can be computed by integral of $v_{t}$ between ranges from zero to time $t$, making time stochastic by the stochastic clock built in. We define the stochastic time change variable $Y_{t}$ within $[0, t]$ as $Y_{t}=\int_{0}^{t} y(\mathrm{~s}) d s$, then the characteristic function of $Y_{t}$ can be computed in the following:

$$
\varphi\left(u, t, y_{0}\right)=\frac{\exp \left(\frac{\kappa^{2} \eta t}{\lambda^{2}}\right)}{\cosh \left(\frac{\gamma t}{2}\right)+\frac{\kappa}{\gamma} \sinh \left(\frac{\gamma t}{2}\right)^{2 \kappa \eta / \lambda^{2}}} \exp \left(y_{0} \frac{2 i u}{\kappa+\operatorname{coth}\left(\frac{\gamma t}{2}\right) \gamma}\right)
$$

with $\gamma=\sqrt{\kappa^{2}-2 \lambda^{2} i u}$.

Then we introduce the tempered stable distributions into the CIR process to formulate time changed TS model process $Z_{t}$ with $Z_{t}=X_{Y_{t}}$ by subordinating the tempered stable distributions to the stochastic volatility process. The stochastic volatility Lévy process accommodates jumps and stochastic volatility simultaneously. When $X_{t}$ follows the NTS process, $Z_{t}$ is the time changed NTS process, the same way to time changed CTS process. Following Schoutens et al. (2003), it is known that the characteristic function of $Z_{t}$ can be calculated as follows:

$$
\varphi_{Z_{t}}(u, t)=\varphi_{Y_{t}}\left(-\mathrm{i} \psi_{X_{t}}(u), t\right)
$$

where $\psi(u)$ denotes the characteristic exponent.

The advantage of stochastic processes to describe stochastic volatility over regime switching method and time series method is that it can be effectively applied in FFT because of the availability of analytical characteristic function form. In this paper, we introduce the stochastic time varying into the tempered stable processes framework by letting the variance of classical tempered stable distribution and normal tempered stable distribution change with time, which method had been used in Carr et al. (2003) for time changed Lévy processes in European option pricing covering VGCIR and NIGCIR models. We extend its framework via incorporating normal tempered stable distribution with random time change to capture the information content of 
markets.

The pure jump infinite activity Lévy process driven stochastic volatility model consists of two parts: one part is a pure jumps Lévy process that controls asset price dynamic evolution process, the other part is a stochastic volatility process that controls the strength of price fluctuations and jump frequency by adjusting the time change rate. The TSSV process is established through subordinating Lévy model to stochastic volatility process which can reflect the jumps behaviors and stochastic volatility of returns $\ln \left(S_{t} / S_{0}\right)$ simultaneously. It not only overcomes shortcomings of pure jump Lévy models that cannot reflect the diffusion process of underlying volatility, but also overcomes the defects of stochastic volatility models that cannot depict infinite jumping behaviors of returns. Then the characteristic function formula of logarithmic returns for TSSV model is derived as follows:

$$
\begin{aligned}
& E\left(e^{i u \ln \frac{S_{t}}{S_{0}}}\right)=\exp \left\{\text { iurt }+\frac{2 \psi(u) Y_{0}\left(1-e^{-\Pi t}\right)}{2 \Pi-(\Pi-\kappa)\left(1-e^{-\Pi t}\right)}-\frac{k \eta}{\lambda^{2}}\left[2 \ln \left[1-\frac{\Pi-\kappa}{2 \Pi}\left(1-e^{-\Pi t}\right)\right]+(\Pi-\kappa) t\right]\right\} \\
& \quad=\exp [\text { iurt }+\tau(t, u)]
\end{aligned}
$$

where $\Pi=\sqrt{\kappa^{2}-2 \psi(u) \sigma^{2}}, \psi(u)$ refers to the characteristic exponent of pure jump Lévy processes, $\kappa, \eta, \lambda$ are parameters of CIR stochastic volatility process.

Then the probability density function can be obtained by fast Fourier transform of characteristic functions. In Fig. 1 we show the effect of the four parameters of CTS marginal distribution in stochastic volatility classical tempered stable model have on density function distribution of CTSSV model, and in Fig. 2 we show the influence of four parameters of NTS marginal distribution in stochastic volatility normal tempered stable model have on the density function distribution of NTSSV model. What can be seen is that parameters $\mathrm{C}_{2}, \alpha$ have inverse relationship with the peaks sharpness of CTSSV model density function, while $\lambda_{ \pm}$affect the overall position of density distribution in CTSSV model, $\theta$ and $v$ are oppositely correlated with the sharpness of the peaks in NTSSV model's density function, $\mu$ influences the overall position of NTSSV density distribution, and $\sigma$ affects the shape of the density function.

The introduction of infinite jump behaviors for underlying asset returns that are modeled by infinite activity Lévy processes can capture asymmetry, leptokurtosis and thicker tail properties in returns in addition to characterizing persistence effect and heteroskedasticity effect in volatility. The Lévy processes driven stochastic volatility models incorporates jumps and stochastic volatility simultaneously, which capture not only jumps but also stochastic volatility in stock price dynamics.
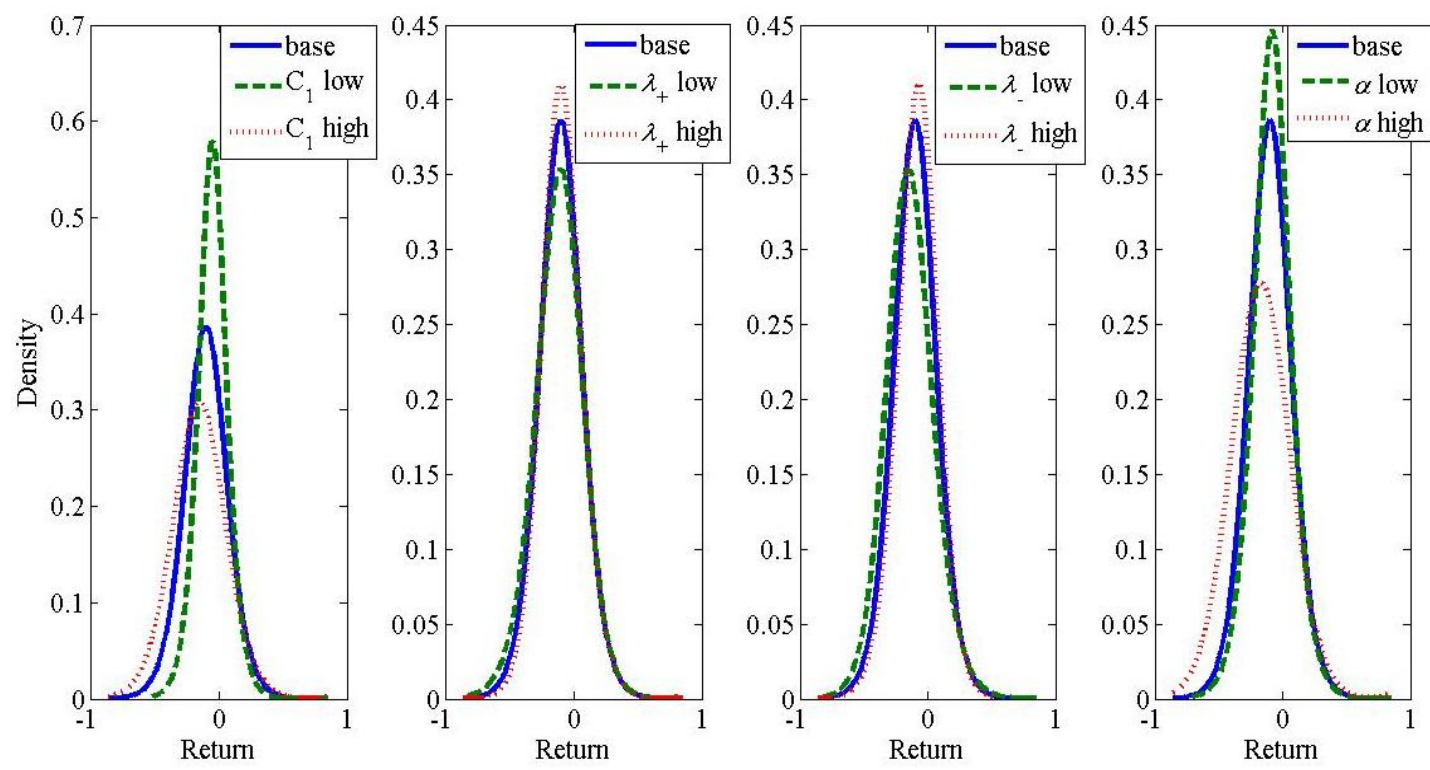

Fig.1. Probability density distribution of CTSSV model 

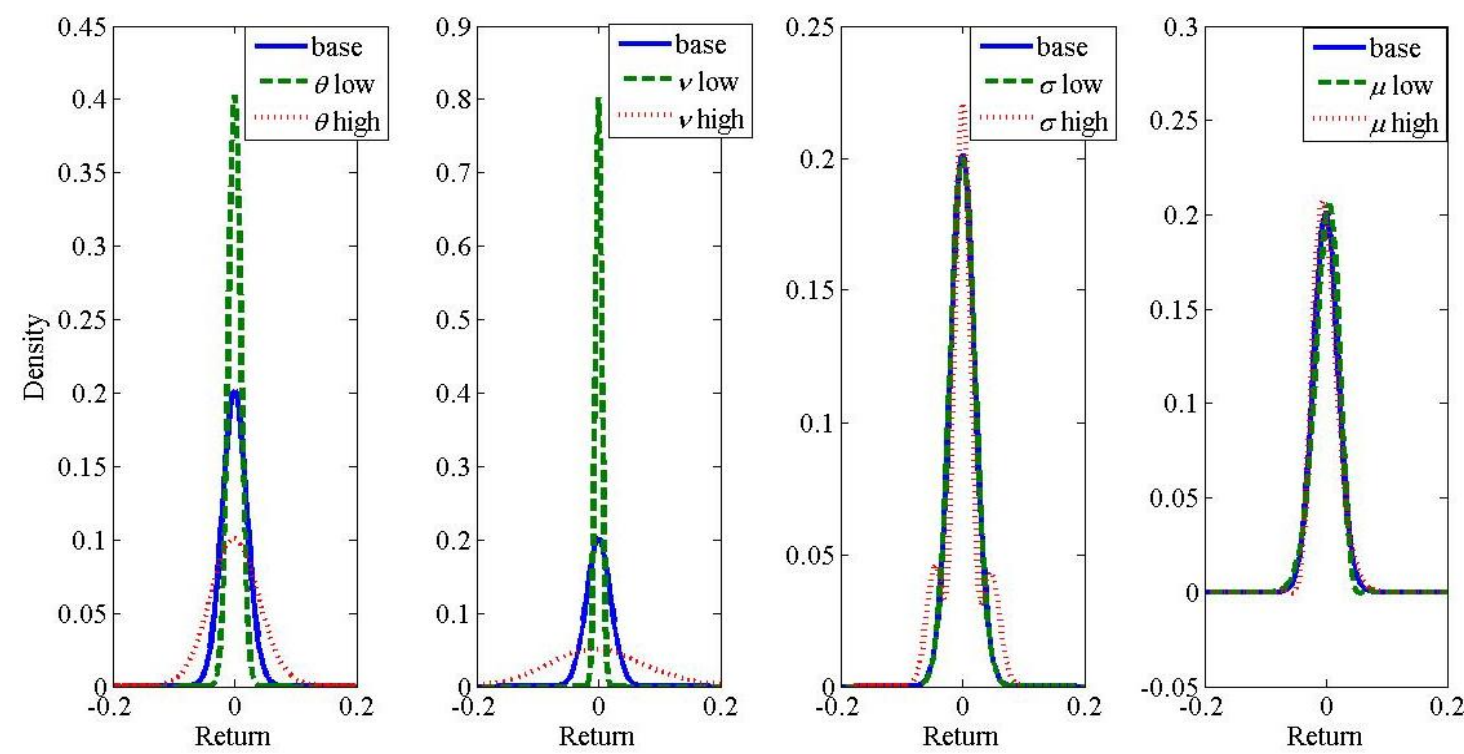

Fig.2. Probability density distribution of NTSSV model

\section{FFT for time changed tempered stable Lévy processes}

\subsection{FFT for $P D F$ and $C D F$}

By utilizing the Fourier transform of the corresponding characteristic functions of time changed tempered stable Lévy processes, analytical expressions of their probability density functions are derived in Menn and Rachev (2006) for $\alpha$ stable distribution, which overcomes the drawbacks of stable distribution that cannot be used for density calculation, and this method is extended to classical tempered stable distribution in Scherer et al. (2012). The availability of closed form characteristic functions of TSSV processes facilitate the application for this class process in measuring market risks and portfolio management. The fast Fourier Transform technique can be utilized to expedite the calculation speed. Hence, the PDF, CDF and VaR as well as CVaR can be derived efficiently utilizing fast Fourier transform method. And the explicit formulas for $\mathrm{VaR}$ and $\mathrm{CVaR}$ are of significant importance in risk assessment and portfolio reversion.

The probability density function $f_{X}$ of random variable $X$ in the TSSV process is calculated from the characteristic function $\varphi(u)$ exploiting the inverse transform of Fourier transform as in formula (12). It is well known that Discrete Fourier transform (DFT) approximate the Fourier transform discretely mapping from vector $\left(x_{1}, \ldots, x_{N}\right)$ to vector $\left(y_{1}, \ldots, y_{N}\right)$ as in formula (13).

$$
\begin{gathered}
f_{X}(x)=\frac{1}{2 \pi} \int_{-\infty}^{+\infty} e^{-i u x} \varphi_{X}(u) d x \\
x_{j}=\sum_{k=1}^{N} y_{k} e^{-i \frac{2 \pi(j-1)(k-1)}{N}}, j=1, \ldots, N
\end{gathered}
$$

The fast Fourier transform can efficiently implement the DFT algorithm, and it approximates the probability density function (PDF) at point $x_{j}$ as follows:

$$
\begin{aligned}
f_{X}\left(x_{j}\right) & \approx \frac{1}{2 \pi} \int_{-a}^{+a} e^{-i u_{j} x} \varphi d u \\
& =\frac{a}{N}(-1)^{i-1} i e^{i \frac{\pi(j-1)}{N}} \mathrm{FF}_{j} \mathrm{~T}+\left(\left(^{(-1} \phi\right) u_{k}{ }^{*}{ }_{k}\left({ }_{1}, N\right) .\right.
\end{aligned}
$$

where $\alpha \in \mathrm{R}$, denotes the integration limits of Fourier transform, $j$ and $k \in\left\{1, \ldots, N=2^{q}\right\}, q \in N$, influences the number of integration steps, $u_{k}=-a+(2 a / N)(k-1), u_{k}{ }^{*}=\left(u_{k+1}+u_{k}\right) / 2, x_{j}=$ $-N \pi / 2 a+(\pi / a)(j-1)$.

The DFT algorithm divides the integration space into equidistant interval of size $2 a / N$, and utilize middle point rule for approximation so as to minimize errors. It can be seen that the choice of $N$ and $a$ is so significant that parameter $a$ affects the sampling distance and interpolation 
interval, and $N$ influences the computational complexity. A reasonable choice is a compromise between sampling errors of interpolation error and discretization error. The property of approximation is determined by the integrand function in each interval.

\subsection{The VaR and CVaR}

It has been illustrated in Kim et al. (2010a) that the CDF $F_{X}=P(X<x)$ of random variable $X$ in the TSSV process can be computed by the following equation with $\rho>0$.

$$
F_{X}(x)=\frac{e^{\rho x}}{\pi} \mathfrak{R}\left\{\int_{0}^{\infty} e^{-i u x} \frac{\varphi_{X}(u+i \rho)}{\rho-i u} d u\right\}
$$

Then, it holds that

$$
\int_{0}^{\infty} e^{-i u x} \frac{\varphi_{X}(u+i \rho)}{\rho-i u} d u \approx \sum_{k=1}^{N} e^{-i u_{k}^{*} x_{j}} \frac{\varphi_{X}\left(u_{k}^{*}+i \rho\right)}{\rho-i u_{k}^{*}} \frac{2 a}{N}
$$

Utilize the calculation equation $x_{j} u_{k}{ }^{*}=-\pi(2 k-1) / 2+(2 \pi / N)((2 k-1) / 2)(j-1)$, the CDF $F_{X}$ can be expressed as follows:

$$
F_{X}(x)=\frac{e^{\rho x}}{\pi} \Re\left(\frac{2 a}{N} e^{-i \frac{\pi}{N}(j-1)} \cdot \operatorname{FFT}_{j}\left((-1)^{k-1} \cdot i \cdot \frac{\varphi_{X}\left(u_{k}^{*}+i \rho\right)}{\rho-i u_{k}^{*}}\right)\right), k=1, \ldots, N
$$

$\mathrm{VaR}_{\delta}$ is defined as the excessive loss with probability $\delta$. The superior risk measure used is the $\mathrm{CVaR}$ that tells how severe the average damage is and is measured by the average of VaRs exceeding VaR. The benefits of employing CVaR over VaR are the inclusion of downside tail in addition to satisfying coherent measure property (Bin, 2015; Gao et al., 2016). Following Kim et al. (2010a), it can be derived that the $\mathrm{VaR}_{\delta}$ at tail probability $\delta$ for returns described by time changed tempered stable processes is expressed by the inverse of cumulative density function of TSSV distributions, namely $\operatorname{VaR}_{\delta}(X)=F_{X}^{-1}(\delta)$. And the analytical formula for $\mathrm{CVaR}_{\delta}$ at confidence level $\delta$ for the TSSV process is calculated as follows:

$$
\mathrm{CVaR}_{\delta}(X)=\operatorname{VaR}_{\delta}(X)-\frac{e^{\mathrm{VaR}_{\delta}(X) \rho}}{\pi(1-\delta)} \mathfrak{R}\left(\int_{0}^{\infty} e^{-i u \mathrm{VaR}_{\delta}(X)} \frac{\varphi_{X}(u+i \rho)}{(u+i \rho)^{2}} d u\right)
$$

\section{Risk-adjusted criteria for stock ranking}

The traditional method to select stocks for portfolio on momentum strategies is to evaluate the realized cumulative returns of individual stocks during a certain period, which is a simple measurement criterion without considering the risk components of underlying stock behaviors. In order to incorporate risk profiles of financial markets, risk measure like variance is implemented to capture excess risk beyond extra returns. However, the deficiency of variance measure with regards to mean-variance framework is that it does not accommodate the investor's attitude towards risk as well as not satisfying coherent risk properties.

Given that the financial returns exhibit leptokurtosis, asymmetry and clustering properties, the risk adjusted criterion for selecting stocks on time varying tempered stable distributions is applied. As an alternative reward risk criterion, CVaR serves as a more conservative measure examining the averaged loss degree exceeds VaR, satisfying properties of monotonicity, subadditivity and positive homogeneity. Under the assumption that stocks returns process has continuous cumulative distribution functions, the CVaR becomes the expected tail loss (ETL) at the corresponding level, namely $\operatorname{CVaR}_{(1-\delta) 100 \%}(r)=\operatorname{ETL}_{\delta 100 \%}(r)$. It has been supported that alternative reward risk criteria on the basis of $\mathrm{CVaR}$ can capture the empirical behaviors of data effectively (Rachev, 2007; Choi, 2015). Hence, we perform the stable tail adjusted return (STAR) ratio and the R ratio proposed in Rachev (2007) so as to reflect the risk profiles and account for stylized facts in addition to Sharpe ratio. We compare these ratios by maximizing the ultimate wealth of portfolio during holding time.

The Sharpe ratio measures the ratio of expected extra return and its corresponding deviation, which can be formulated as follows:

$$
\text { Sharpe }=\frac{E\left(r-r_{f}\right)}{\sigma\left(r-r_{f}\right)}
$$

where $r_{f}$ denotes the risk free rate, $\sigma$ in denominator represents standard deviation. It considers 
both downside deviation and upward deviation that may not be true risk actually. The portfolio with larger Sharpe ratio means higher returns per deviation. When assuming non-Gaussian distribution for returns process, the portfolio consequences using Sharpe ratio may be unreliable.

The $\operatorname{STAR}_{(1-\delta) 100 \%}$ ratio focusing on downside risk substitutes the standard deviation in Sharpe ratio into the conditional value at risk in denominator, it can be expressed as follows:

$$
\operatorname{STAR}_{(1-\delta) 100 \%}=\frac{E\left(r-r_{f}\right)}{\operatorname{CVaR}_{(1-\delta) 100 \%}\left(r-r_{f}\right)}
$$

The different choices of significance level $\delta$ in $\operatorname{STAR}_{(1-\delta) 100 \%}$ ratio signify different risks degree for downside risks, representing the attitudes towards risks of investors. The higher the value of $\delta$ is, the more aversion investors exhibit.

The $\mathbf{R}_{(\alpha, \beta)}$ ratio applied to different significance levels for bilateral tail distributions is the ratio between $\mathrm{ETL}_{\alpha 100 \%}$ of inverse excess returns and $\mathrm{ETL}_{\beta 100 \%}$ of extra returns. It measures expected upside tail reward and downward tail loss and can be expressed as follows:

$$
\mathrm{R}_{(\alpha, \beta)}=\frac{\operatorname{ETL}_{\alpha 100 \%}\left(r_{f}-r\right)}{\operatorname{ETL}_{\beta 100 \%}\left(r-r_{f}\right)}
$$

where $\alpha$ and $\beta$ denote different significance levels for right and left tails. This ratio simultaneously measures expected right tail rewards exceeding $\alpha \%$ level and expected right tail losses exceeding $\beta \%$ level, awarding excessive returns for excessive losses. However, it does not consider significance levels between $\alpha$ and $\beta$. What can also be concluded is that $\operatorname{STAR}_{(1-\delta) 100 \%}$ ratio and $\mathbf{R}_{(\alpha \text {, }}$ $\beta$ ) ratio can evaluate portfolio returns that display heavy tails.

\section{Empirical research}

\subsection{Parameter estimation}

We estimate parameters for VGCIR model, CTSSV model and NTSSV model to investigate the forecasting performance from January 3, 2008 to December 31, 2015, in which period Chinese financial market experiences two meltdowns in year 2008 and year 2015, respectively. Each daily estimation values are obtained using 5 years historical returns data of Hangseng index (HSI) before the selected day in Chinese Hong Kong market. And the value of the risk-free rate of return is the 90 day benchmark deposit rate issued by the central bank of China. Fig. 3 displays the returns series, volatility series, skewness sequences and kurtosis changes sequences of HSI. What can be seen from Fig. 3 is that Hangseng index displays irregular infinite jumps in stock prices dynamics as well as exhibiting volatility clustering and persistence in series in addition to mean reversion tendency. The skewness and kurtosis changes show leptokurtosis and left skew phenomenon in returns. The daily amplitude of four statistics varies over time. We employ maximum likelihood estimation method for parameters estimation, and list the results in Table 1. In order to assess the goodness of fit and evaluate the tail fit, the Kolmogorov Smirnov (KS) statistic as well as the corresponding $p$ values and Anderson-Darling (AD) statistic are computed.

Table 1

\begin{tabular}{|c|c|c|c|c|c|c|c|c|c|}
\hline Model & & & & arameter & & & & $\begin{array}{c}\mathrm{KS} \\
(p \text { value })\end{array}$ & $\mathrm{AD}$ \\
\hline VGCIR & $\begin{array}{c}\mathrm{C}_{1} \\
14.5429\end{array}$ & $\begin{array}{c}\mathrm{G} \\
18.2635\end{array}$ & $\begin{array}{c}\mathrm{M} \\
23.1738\end{array}$ & $\begin{array}{c}\kappa \\
9.5438\end{array}$ & $\begin{array}{c}\eta \\
0.6547\end{array}$ & $\begin{array}{c}\lambda \\
0.6283\end{array}$ & & $\begin{array}{c}0.0437 \\
(0.0039)\end{array}$ & 0.3674 \\
\hline CTSSV & $\begin{array}{c}\mathrm{C}_{2} \\
6.0066\end{array}$ & $\begin{array}{c}\lambda \\
16.5312\end{array}$ & $\begin{array}{c}\lambda_{+} \\
28.7948\end{array}$ & $\begin{array}{c}\alpha \\
0.7264\end{array}$ & $\begin{array}{c}\kappa \\
7.3655\end{array}$ & $\begin{array}{c}\eta \\
0.4218\end{array}$ & $\begin{array}{c}\lambda \\
0.8973\end{array}$ & $\begin{array}{c}0.0423 \\
(0.0995)\end{array}$ & 0.1233 \\
\hline NTSSV & $\begin{array}{c}\mu \\
-0.0728\end{array}$ & $\begin{array}{c}v \\
15.3949\end{array}$ & $\begin{array}{c}\sigma \\
0.0623\end{array}$ & $\begin{array}{c}\theta \\
0.6743\end{array}$ & $\begin{array}{c}\kappa \\
7.8994\end{array}$ & $\begin{array}{c}\eta \\
0.3645\end{array}$ & $\begin{array}{c}\lambda \\
0.9106\end{array}$ & $\begin{array}{c}0.0325 \\
(0.1782)\end{array}$ & 0.0915 \\
\hline
\end{tabular}

Estimation results

It can be concluded from Table 1 that both the three models are accepted by KS test at the $1 \%$ significance level in addition to AD test, which exhibits superiority modeling ability of time changed tempered stable Lévy processes. Moreover, the $p$ values of these models are all larger than $1 \%$. It is obvious that the AD statistic of VGCIR model is larger than that of time changed tempered stable models, meaning that the TSSV models can better describe the fat tail behaviors of empirical distribution. Seen from the statistics, the NTSSV model fits the data best and seems most appropriate for market data. The negative value of $\mu$ in NTSSV model indicates left skewness of HSI returns. The consequences of CTSSV distribution with $\lambda_{+}=29.7948>\lambda_{-}=$ 
16.5312 validates the left skewed returns distribution. The value of shape parameter $\alpha$ in CTSSV model indicates leptokurtic nature and fat tail phenomenon in returns. When we look at the volatility diffusion components, the parameter values of volatility reverting rate $\kappa$ and variance of volatility $\eta$ in TSSV models are smaller than those of VGCIR model. This may mainly be caused by the strong power of tempered stable distributions in capturing infinite activity properties in returns and thus decreasing the persistence of the volatility diffusion components.
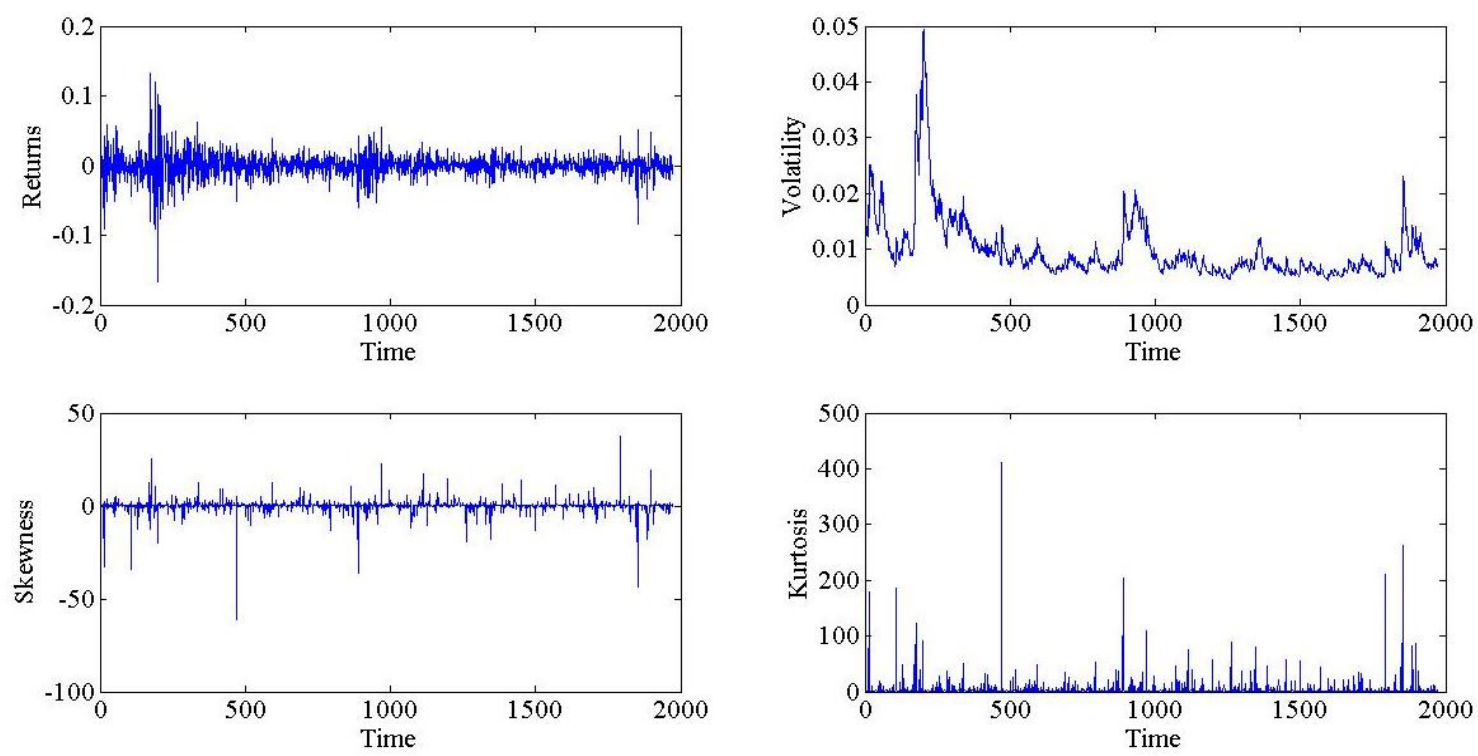

Fig.3. Hangseng daily index statistics

\subsection{Backtesting for VaR and CVaR}

We calculate the 99\%VaR and 99\%CVaR for HSI using VGCIR model, CTSSV model and NTSSV model, and display them in Fig.4. In order to evaluate the accuracy of forecasting, the backtesting consisting of the Christoffersen likelihood ratio (CLR) test and the Berkowitz likelihood ratio (BLR) test for different models are performed. The whole data for backtesting are divided into three periods, which are two volatile periods consisting of years from 2008 to 2009 and year 2015, one relatively calm period including 2010 to 2014. The interval based CLR test counts violations when losses in reality exceed the forecasted VaR. It comprises the CLR test of unconditional coverage $\left(\mathrm{CLR}_{\mathrm{uc}}\right)$, the CLR test of independence $\left(\mathrm{CLR}_{\mathrm{ind}}\right)$, the joint test of coverage and independence $\left(\mathrm{CLR}_{\mathrm{cc}}\right)$. The density based BLR test calculates the inverse function of cumulative distribution function to implement test of independence $\left(B^{2} R_{\text {ind }}\right)$ besides assessing the accuracy of tail distribution forecast $\left(\mathrm{BLR}_{\text {tail }}\right)$. In BLR test, we consider the forecasted CVaR to the realized CVaR. In Table 2, two statistics and the corresponding $p$ values are listed for three time periods.

Table 2

Backtesting for forecasting

\begin{tabular}{|c|c|c|c|c|c|c|}
\hline \multirow[t]{2}{*}{ Model } & \multicolumn{6}{|c|}{ January 5, 2008 to December 31, 2009} \\
\hline & $\begin{array}{c}\mathrm{CLR}_{\mathrm{uc}} \\
\text { ( } p \text { value })\end{array}$ & $\begin{array}{l}\mathrm{CLR}_{\text {ind }} \\
\text { ( } p \text { value) }\end{array}$ & $\begin{array}{c}\mathrm{CLR}_{\mathrm{cc}} \\
(p \text { value })\end{array}$ & $\begin{array}{c}\mathrm{BLR}_{\text {ind }} \\
(p \text { value })\end{array}$ & $\begin{array}{c}\mathrm{BLR}_{\text {tail }} \\
\text { ( } p \text { value })\end{array}$ & Violation \\
\hline VGCIR & $\begin{array}{l}15.8247 \\
(\mathbf{0 . 0 0 0 4})\end{array}$ & $\begin{array}{c}0.0571 \\
(0.8352)\end{array}$ & $\begin{array}{l}20.3046 \\
(\mathbf{0 . 0 0 0 3})\end{array}$ & $\begin{array}{c}4.0517 \\
(0.0411)\end{array}$ & $\begin{array}{l}17.6723 \\
(\mathbf{0 . 0 0 0 5})\end{array}$ & 12 \\
\hline CTSSV & $\begin{array}{l}12.5477 \\
(\mathbf{0 . 0 0 0 5})\end{array}$ & $\begin{array}{c}0.7381 \\
(0.7879)\end{array}$ & $\begin{array}{c}8.4326 \\
(0.0170)\end{array}$ & $\begin{array}{c}3.8574 \\
(0.0472)\end{array}$ & $\begin{array}{l}13.1422 \\
(\mathbf{0 . 0 0 3 7})\end{array}$ & 11 \\
\hline NTSSV & $\begin{array}{c}5.1436 \\
(0.0152) \\
\end{array}$ & $\begin{array}{c}0.0526 \\
(0.8349) \\
\end{array}$ & $\begin{array}{r}9.1433 \\
(0.0114) \\
\end{array}$ & $\begin{array}{c}2.9781 \\
(0.0849) \\
\end{array}$ & $\begin{array}{c}7.2662 \\
(0.0405) \\
\end{array}$ & 9 \\
\hline Model & \multicolumn{6}{|c|}{ January 5, 2010 to December 31, 2014} \\
\hline & $\begin{array}{c}\mathrm{CLR}_{\mathrm{uc}} \\
(p \text { value })\end{array}$ & $\begin{array}{c}\mathrm{CLR}_{\text {ind }} \\
(p \text { value })\end{array}$ & $\begin{array}{c}\mathrm{CLR}_{\mathrm{cc}} \\
(p \text { value })\end{array}$ & $\begin{array}{c}\mathrm{BLR}_{\text {ind }} \\
(p \text { value })\end{array}$ & $\begin{array}{c}\mathrm{BLR}_{\text {tail }} \\
(p \text { value })\end{array}$ & Violation \\
\hline VGCIR & $\begin{array}{c}2.5429 \\
(0.1537)\end{array}$ & $\begin{array}{c}1.7225 \\
(0.1579)\end{array}$ & $\begin{array}{l}11.1345 \\
(\mathbf{0 . 0 0 4 1})\end{array}$ & $\begin{array}{c}1.3226 \\
(0.2475)\end{array}$ & $\begin{array}{l}11.0142 \\
(\mathbf{0 . 0 0 5 1})\end{array}$ & 10 \\
\hline CTSSV & $\begin{array}{c}2.3617 \\
(0.1549)\end{array}$ & $\begin{array}{c}0.3546 \\
(0.5341)\end{array}$ & $\begin{array}{c}9.1703 \\
(0.0002)\end{array}$ & $\begin{array}{c}0.9536 \\
(0.3266)\end{array}$ & $\begin{array}{c}9.8824 \\
(\mathbf{0 . 0 0 8 9})\end{array}$ & 10 \\
\hline
\end{tabular}




\begin{tabular}{|c|c|c|c|c|c|c|}
\hline NTSSV & $\begin{array}{c}2.4788 \\
(0.1541) \\
\end{array}$ & $\begin{array}{c}0.9468 \\
(0.3625) \\
\end{array}$ & $\begin{array}{c}8.2146 \\
(0.0168)\end{array}$ & $\begin{array}{c}0.8024 \\
(0.3695)\end{array}$ & $\begin{array}{c}7.3824 \\
(0.0398)\end{array}$ & 8 \\
\hline Model & \multicolumn{6}{|c|}{ January 5,2015 to December 31,2015} \\
\hline & $\begin{array}{c}\mathrm{CLR}_{\mathrm{uc}} \\
(p \text { value })\end{array}$ & $\begin{array}{c}\mathrm{CLR}_{\text {ind }} \\
(p \text { value })\end{array}$ & $\begin{array}{c}\mathrm{CLR}_{\mathrm{cc}} \\
(p \text { value })\end{array}$ & $\begin{array}{c}\mathrm{BLR}_{\text {ind }} \\
(p \text { value })\end{array}$ & $\begin{array}{c}\mathrm{BLR}_{\text {tail }} \\
(p \text { value })\end{array}$ & Violation \\
\hline VGCIR & $\begin{array}{c}8.3243 \\
(\mathbf{0 . 0 0 2 4})\end{array}$ & $\begin{array}{c}0.4336 \\
(0.5001)\end{array}$ & $\begin{array}{l}16.3964 \\
(0.0005)\end{array}$ & $\begin{array}{c}4.0434 \\
(0.0586)\end{array}$ & $\begin{array}{l}13.4725 \\
(\mathbf{0 . 0 0 1 7})\end{array}$ & 5 \\
\hline CTSSV & $\begin{array}{c}7.3615 \\
(\mathbf{0 . 0 0 6 5})\end{array}$ & $\begin{array}{c}0.7381 \\
(0.3994)\end{array}$ & $\begin{array}{c}9.2936 \\
(0.0102)\end{array}$ & $\begin{array}{c}2.8761 \\
(0.0873)\end{array}$ & $\begin{array}{l}10.5899 \\
(\mathbf{0 . 0 0 3 6})\end{array}$ & 6 \\
\hline NTSSV & $\begin{array}{c}5.3957 \\
(0.0183) \\
\end{array}$ & $\begin{array}{c}0.3962 \\
(0.5328) \\
\end{array}$ & $\begin{array}{c}8.3858 \\
(0.0162) \\
\end{array}$ & $\begin{array}{c}3.0015 \\
(0.0788) \\
\end{array}$ & $\begin{array}{c}8.2434 \\
(0.0157) \\
\end{array}$ & 4 \\
\hline
\end{tabular}

$p$ values in bold indicate rejection of the null hypothesis.
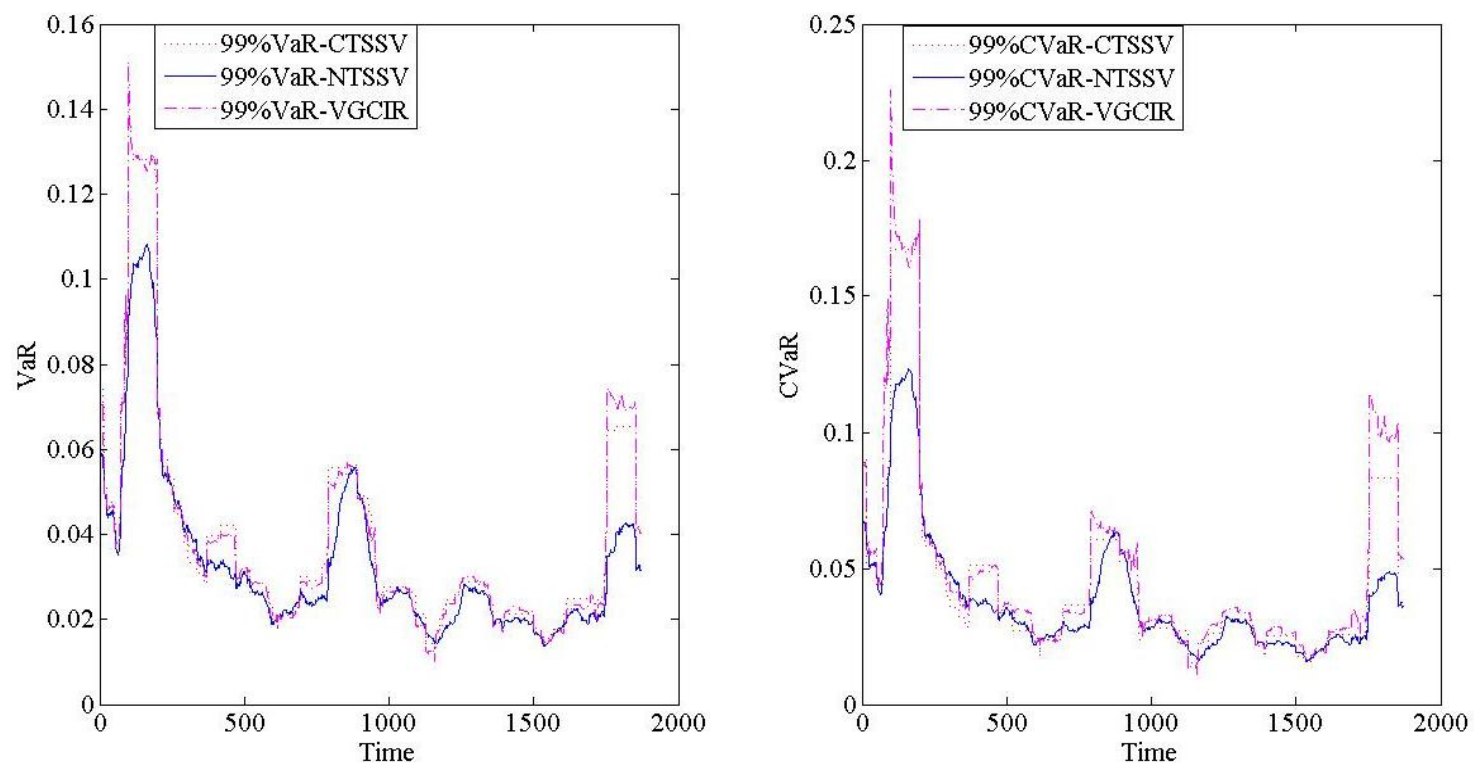

Fig.4. VaR and CVaR for time changed Lévy models

It can be concluded from the above table that for two volatile periods, the VGCIR model is rejected for both CLR test and BLR test in $\mathrm{CLR}_{\mathrm{uc}}, \mathrm{CLR}_{\mathrm{cc}}$ and $\mathrm{BLR}_{\text {tail }}$ statistics at $1 \%$ significance

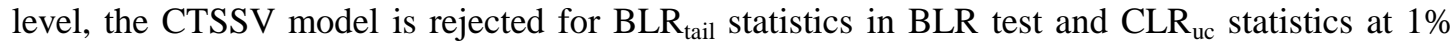
significance level, with the NTSSV model passing all the statistic tests in CLR test and BLR test. All time changed Lévy process models are accepted by the independence examinations of CLR ind $_{\text {in }}$ test and BLR ind test. What can be seen from the consequences of the BLR ${ }_{\text {tail }}$ test of the tail distribution is that CVaR utilizing the NTSSV model is not rejected for the three time periods. During the relatively calm period for 2010 to 2014, the VGCIR model is rejected in CLR $_{\mathrm{cc}}$ test and $\mathrm{BLR}_{\text {tail }}$ test at $1 \%$ significance level, the CTSSV model is rejected in $\mathrm{BLR}_{\text {tail }}$ test, the differences of backtesting performance between CTSSV model and NTSSV model are not obvious, with only NTSSV model passing both the CLR test and BLR test. It is the NTSSV distribution that is most suitable in financial risk measurement.

\subsection{Summary statistics of portfolio under TSSV}

For the portfolio strategy, we employ the sample of 50 stocks in the HSI universe with the same time length as backtesting. The sample stock is ranked during a ranking period for six months before the equally weighted portfolios are evaluated during the holding period for the subsequent six months. The total stocks are divided into 10 equally weighted sub-portfolios, the Group $_{\text {good }}$ stand for the top $10 \%$ of the stocks portfolios with highest values based on the risk adjusted criteria, and Group bad stand for the last $10 \%$ of the stocks portfolios with lowest values based on the risk adjusted criteria. In Fig.5, we give the plot of mean-variance frontier, mean-VaR frontier and mean-CVaR frontier of the Group good portfolio utilizing NTSSV distribution. And summary statistics about portfolio strategies using stochastic volatility tempered stable models based on Sharpe ratio, STAR $_{99 \%}$ ratio and $\mathrm{R}_{99 \%, 99 \%}$ ratio calculation criterions are listed in Table 3 . It includes mean, deviation, skewness, kurtosis and final wealth, which are the obtained ultimate total cumulative returns for portfolios with unit initial wealth. 
Table 3

Portfolio returns and summary statistics

\begin{tabular}{|c|c|c|c|c|c|c|c|}
\hline \multirow{8}{*}{$\frac{\text { Model }}{\text { CTSSV }}$} & Ratio & Portfolio & \multicolumn{5}{|c|}{ Summary statistics } \\
\hline & & & Mean & Deviation & Skewness & Kurtosis & $\begin{array}{c}\text { Final } \\
\text { wealth }\end{array}$ \\
\hline & $\begin{array}{l}\text { Sharpe } \\
\text { ratio }\end{array}$ & Group $_{\text {good }}$ & 0.0003 & 0.0305 & -0.0207 & 6.1746 & 5.1536 \\
\hline & & Group $_{\text {bad }}$ & -0.0001 & 0.0314 & -0.0163 & 7.3288 & 0.2805 \\
\hline & $\begin{array}{l}\text { STAR } \\
(99 \%)\end{array}$ & Group $_{\text {good }}$ & 0.0002 & 0.0292 & -0.0353 & 6.6571 & 5.8269 \\
\hline & & Group $_{\text {bad }}$ & -0.0002 & 0.0305 & -0.0258 & 7.7463 & 0.5531 \\
\hline & R ratio & Group $_{\text {good }}$ & 0.0001 & 0.0291 & -0.0174 & 6.3304 & 5.2174 \\
\hline & & Group $_{\text {bad }}$ & -0.0002 & 0.0303 & -0.0165 & 7.5284 & 0.4825 \\
\hline NTSSV & & & & & & & \\
\hline & $\begin{array}{l}\text { Sharpe } \\
\text { ratio }\end{array}$ & Group $_{\text {good }}$ & 0.0004 & 0.0293 & -0.0188 & 6.4531 & 4.8234 \\
\hline & & Group $_{\text {bad }}$ & 0.0001 & 0.0295 & -0.0143 & 7.1544 & 0.2903 \\
\hline & $\begin{array}{l}\text { STAR } \\
(99 \%)\end{array}$ & Group $_{\text {good }}$ & 0.0002 & 0.0291 & -0.0315 & 6.1256 & 6.1273 \\
\hline & & Group $_{\text {bad }}$ & -0.0002 & 0.0284 & -0.0297 & 7.3201 & 0.4684 \\
\hline & R ratio & Group $_{\text {good }}$ & 0.0002 & 0.0291 & -0.0152 & 6.6094 & 5.6108 \\
\hline & & Group $_{\mathrm{bad}}$ & -0.0001 & 0.0282 & -0.0139 & 7.3953 & 0.4112 \\
\hline
\end{tabular}

Several findings can be found from the above table. The largest daily mean value of 0.0004 for all reward risk ratios appears in the Sharpe ratio for NTSSV model, followed by the result of Sharpe ratio appears in CTSSV model, with the STAR ratio achieving the lowest average reward

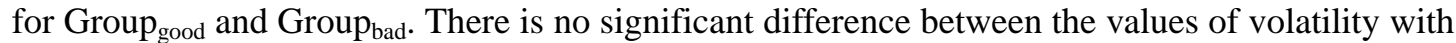

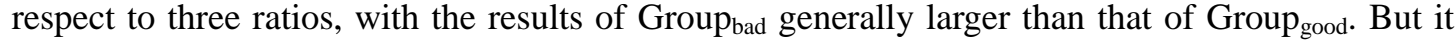
can be obviously seen that $\mathrm{R}_{99 \%, 99 \%}$ ratios obtain the lowest values of deviation compared with other two ratios, and the CVaR based alternative rules not only get lower volatility but also outperform others. Although the Sharpe ratio performs slightly poorer, its deviation is reduced using NTSSV distribution than CTSSV. Besides, the estimations of Sharpe ratio are slightly higher than using other alternative ratios, suggesting that strategies employing variance as risk indicators is risker than that employing conditional value at risk as indicators. The lowest magnitude of

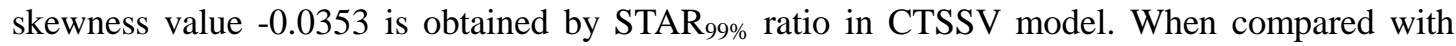

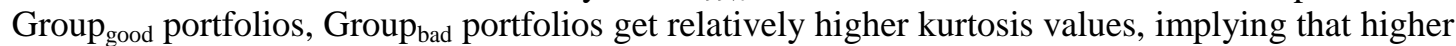
reward strategies are associated with lower skewness. The similar consequences can be found in kurtosis estimations that larger kurtosis values arise in Group bad portfolio for all the three reward risk ratios. From the final wealth of observation period for our strategies, it can be seen that all the three reward risk ratios obtain positive profits. Among all the criteria, the largest value of 6.1273 arises in STAR $_{99 \%}$ ratio of NTSSV distribution, followed by $\mathrm{R}_{99 \%, 99 \%}$ ratio with a value of 5.6108. The smallest results for ultimate wealth of portfolio employing TSSV models are obtained for the Sharpe ratio with values of 0.2805 and 0.2903 . These consequences indicate that strategies using $\mathrm{CVaR}$ as risk measurement obtain better performance accepting lower tail risks.

\subsection{Distributional analysis of portfolio under TSSV}

Empirical evidence has supported the fact that asset returns do not display normal distribution with excessive peak, fat tail and asymmetric nature. The construction of time changed tempered stable Lévy processes provides a good modeling framework for portfolio theory. The portfolio deciles (Group $_{\text {good }}$ and Group $_{\text {bad }}$ ) attained on different stock selection criteria are investigated about how portfolio returns associate with parameters estimations of time changed tempered stable distributions. In order to investigate the fitting performance of different Lévy based stochastic volatility processes, Kolmogorov distance (KD) statistic that measures the distance between realized sample distribution and cumulative density function of estimated density is employed to examine which model is closer to empirical returns. The estimated results for portfolio are listed in Table 4.

Table 4

Portfolio estimation results 


\begin{tabular}{|c|c|c|c|c|c|c|c|c|c|c|}
\hline \multirow{2}{*}{$\begin{array}{c}\text { Model } \\
\text { VGCI } \\
\text { R }\end{array}$} & \multirow[t]{2}{*}{ Ratio } & \multirow[t]{2}{*}{ Portfolio } & \multicolumn{7}{|c|}{ Parameter } & \multirow[t]{2}{*}{$\mathrm{KD}$} \\
\hline & & & $\mathrm{C}_{1}$ & $\mathrm{G}$ & $\mathrm{M}$ & $\kappa$ & $\eta$ & $\lambda$ & & \\
\hline & Sharpe & Group $_{\text {goo }}$ & 12.562 & 12.689 & 20.357 & 9.877 & 0.753 & 0.511 & & 0.041 \\
\hline & ratio & 1 govo & 3 & 4 & 6 & 4 & 9 & 7 & & 6 \\
\hline & & Group $_{\text {bad }}$ & 13.463 & 12.147 & 20.468 & 9.884 & 0.749 & 0.533 & & 0.037 \\
\hline & & & 9 & 6 & 2 & 7 & 8 & 4 & & 4 \\
\hline & STAR & Group $_{\text {goo }}$ & 15.100 & 13.226 & 17.449 & 9.256 & 0.700 & 0.496 & & 0.038 \\
\hline & $(99 \%)$ & $\mathrm{d}^{2}$ & 4 & 1 & 5 & 2 & 2 & 7 & & 1 \\
\hline & & Group $_{b a d}$ & 14.873 & 11.176 & 18.072 & 9.273 & 0.713 & 0.523 & & 0.038 \\
\hline & & & 7 & 9 & 6 & 8 & 4 & 8 & & 8 \\
\hline & $\mathrm{R}$ ratio & Group $_{\text {goo }}$ & 9.5812 & 10.131 & 17.257 & 9.001 & 0.728 & 0.586 & & 0.038 \\
\hline & $(99 \%, 99 \%$ & d & & 8 & 7 & 3 & 5 & 1 & & 5 \\
\hline & & Group $_{\text {bad }}$ & 11.305 & 9.7562 & 17.396 & 9.568 & 0.734 & 0.564 & & 0.038 \\
\hline & & & 8 & & 6 & 2 & 0 & 9 & & 9 \\
\hline \multirow{13}{*}{$\begin{array}{c}\text { CTSS } \\
\mathrm{V}\end{array}$} & & & $\mathrm{C}_{2}$ & $\lambda_{-}$ & $\lambda_{+}$ & $\alpha$ & $\kappa$ & $\eta$ & $\lambda$ & KD \\
\hline & Sharpe & Group $_{\sigma}$ & 4.7925 & 13.875 & 25.172 & 0.726 & 7.595 & 0.511 & 0.739 & 0.028 \\
\hline & ratio & $\mathrm{d}$ & & 1 & 3 & 7 & 7 & 4 & 1 & 5 \\
\hline & & Group $_{\mathrm{bad}}$ & 4.8022 & 14.994 & 24.330 & 0.617 & 7.552 & 0.500 & 0.741 & 0.025 \\
\hline & & & & 2 & 5 & 2 & 4 & 2 & 0 & 6 \\
\hline & STAR & Group $_{\text {goo }}$ & 5.2581 & 12.397 & 27.845 & 0.793 & 7.698 & 0.538 & 0.773 & 0.030 \\
\hline & $(99 \%)$ & 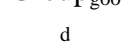 & & 4 & 3 & 6 & 9 & 6 & 2 & 1 \\
\hline & & Group $_{b a d}$ & 6.6549 & 11.546 & 23.296 & 0.523 & 7.521 & 0.511 & 0.783 & 0.029 \\
\hline & & & & 3 & 2 & 4 & 2 & 3 & 5 & 6 \\
\hline & $\mathrm{R}$ ratio & Group $_{\text {goo }}$ & 5.1920 & 15.775 & 27.954 & 0.840 & 7.623 & 0.596 & 0.695 & 0.031 \\
\hline & $(99 \%, 99 \%$ & $\mathrm{d}$ & & 1 & 5 & 5 & 4 & 2 & 4 & 1 \\
\hline & & Group $_{\text {bad }}$ & 5.7433 & 16.124 & 26.308 & 0.816 & 7.054 & 0.567 & 0.703 & 0.028 \\
\hline & & & & 5 & 8 & 9 & 8 & 7 & 3 & 7 \\
\hline \multirow{13}{*}{$\begin{array}{c}\text { NTSS } \\
\mathrm{V}\end{array}$} & & & $\mu$ & $v$ & $\sigma$ & $\theta$ & $\kappa$ & $\eta$ & $\lambda$ & KD \\
\hline & Sharne & Groun & -00329 & 15,411 & 0.0514 & 0.726 & 7.750 & 0.438 & 0.826 & 0021 \\
\hline & ratio & Uivergoo & & 5 & & 7 & 1 & 1 & 5 & 5 \\
\hline & & Group $_{\text {bad }}$ & -0.0416 & 17.283 & 0.0309 & 0.713 & 7.629 & 0.420 & 0.814 & 0.019 \\
\hline & & & & 8 & & 8 & 5 & 9 & 9 & 7 \\
\hline & STAR & Group $_{\text {goo }}$ & -0.0426 & 14.235 & 0.0692 & 0.873 & 7.881 & 0.445 & 0.883 & 0.014 \\
\hline & $(99 \%)$ & d & & 8 & & 6 & 6 & 5 & 7 & 2 \\
\hline & & Group $_{\text {bad }}$ & -0.0574 & 16.883 & 0.0755 & 0.554 & 7.646 & 0.372 & 0.856 & 0.015 \\
\hline & & & & 1 & & 1 & 6 & 1 & 2 & 6 \\
\hline & $\mathrm{R}$ ratio & Group $_{\text {goo }}$ & -0.0689 & 15.974 & 0.0545 & 0.664 & 7.825 & 0.495 & 0.844 & 0.022 \\
\hline & $(99 \%, 99 \%$ & d & & 7 & & 8 & 5 & 7 & 1 & 3 \\
\hline & & Group $_{b a d}$ & -0.0763 & 18.523 & 0.0561 & 0.512 & 7.012 & 0.423 & 0.825 & 0.017 \\
\hline & & & & 9 & & 5 & 4 & 6 & 6 & 5 \\
\hline
\end{tabular}

In VGCIR model, parameters $\mathrm{G}_{1}$ and $\mathrm{M}$ influence the arrival rates of jumps in upward and downward directions, respectively. The values of $\mathrm{G}_{1}$ for group $\mathrm{pad}_{\mathrm{b}}$ are lower than that of group $\mathrm{g}_{\mathrm{good}}$, while values of $\mathrm{M}$ for group $\mathrm{bad}_{\mathrm{b}}$ are higher than that of group $\mathrm{p}_{\text {good }}$. The scale parameter $\mathrm{C}_{2}$ in CTSSV model associates with kurtosis of the density function, with smaller $\mathrm{C}_{2}$ resulting to higher peak. For Sharpe ratio, STAR ratio, and $\mathrm{R}$ ratio, the estimates of $\mathrm{C}_{2}$ in group $\mathrm{p}_{\mathrm{bad}}$ are higher than that in

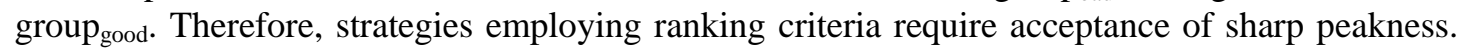
Additionally, the values of shape parameter $\alpha$ in CTSSV model have contrary relationship with the sharpness of peak of CTSSV density functions, namely, $\alpha$ relates to leptokurtic and fat tail nature in returns. For the portfolio returns of extreme deciles, the estimated values of shape parameter $\alpha$ are in the range $(0.7267,0.8405)$ for group good $_{\text {deciles and in the range }(0.5234,0.8169) \text { for }}$ group $_{\text {bad }}$ deciles, which suggests that strategies with higher mean are associated with higher scale parameters. The fact that the values of $\alpha$ are all below 2 suggests that Gaussian distribution is not appropriate in describing portfolio returns. Since the highest value of $\alpha$ for extreme deciles appears in $\mathrm{R}$ ratio among all ranking ratios, it indicates that the $\mathrm{R}$ ratio reduces tail risk by accepting less fat tail distributions, turning into the most effective criteria for selecting stocks. Parameters $\lambda_{+}$and $\lambda_{-}$in CTSSV distribution grasp tails decay rate in positive and negative sides, respectively. If $\lambda_{+}$disagrees with $\lambda_{-}$, it is asymmetric in jumps structure. From all the three ratios, 
there exists apparent left skewness with $\lambda_{+}>\lambda_{-}$. Additionally, $\lambda_{-}$values for $\mathrm{R}_{99 \%, 99 \%}$ ratio are higher than those of other rules, which indicates that portfolios constructed under $\mathbf{R}_{99 \%, 99 \%}$ ratio are exposed to lower tail risks, or the strategy is skilled in controlling downside risks. Since the $\mathrm{R}$ ratio considers both the upward rewards and downward risks, it can better construct portfolios in risk management.

Moreover, parameter $\mu$ in NTSSV distribution reflects the skewness of density function. The negative estimations consequences from both group ${ }_{\text {good }}$ deciles and group bad $_{\text {deciles confirm the }}$ asymmetry of financial data, showing that strategies employing Sharpe ratio, STAR ratio, and R ratio as ranking criteria accommodate negative skewness. Since higher kurtosis in time series corresponds to higher values of $\theta$. Furthermore, $\theta$ and $v$ are oppositely correlated with the sharpness of the peaks in NTSSV model's density function. What can be concluded from above is that higher mean strategy is related to lower kurtosis. Then we look at the volatility diffusion components using different ranking criteria, the values of volatility reverting rate $\kappa$ and variance of volatility $\eta$ in TSSV models are smaller than those of VGCIR model, consistent with the results obtained previously. While they are relatively higher in group good $_{\text {than }}$ that in group bad $_{\text {for TSSV }}$ models except some outliers with STAR ratio and $\mathrm{R}$ ratio getting higher values compared to that of Sharpe ratio. Additionally, it can be inferred from the KD statistic that NTSSV model is superior to CTSSV model and VGCIR model in describing portfolio returns dynamics, providing a better theoretical model for portfolio theory.

\section{Conclusion}

Abundant evidence shows that financial returns display leptokurtosis, asymmetry, clustering properties and heteroskedasticity effect. In this paper, we subordinate normal tempered stable processes to time varying stochastic volatility processes to construct time changed tempered stable models framework and apply it in risk management. We accommodate stochastic volatility and Lévy jumps in asset returns simultaneously through subordinating tempered stable processes to the stochastic volatility models, which reflect the abnormality behaviors in returns and clustering effect in stochastic volatility. Through exploiting Fourier transform technique and characteristic functions for probability calculation, the analytical formulas of $\mathrm{VaR}$ and $\mathrm{CVaR}$ are derived.

Moreover, we measure the financial risk of Haneseng index by computing VaR and CVaR using time changed tempered stable processes to capture stylized facts. By conducting researches on backtesting for $\mathrm{VaR}$ and $\mathrm{CVaR}$, it leads to conclusion that the NTSSV distribution has better risk predictive power in measuring risk, and is most suitable in financial risk measurement. Furthermore, we investigate portfolio performance applying alternative risk adjusted stock selection criteria for portfolio strategies in various assets distributions. The portfolio performance observation indicates that strategies using CVaR as risk measurement obtain better performance accepting lower tail risks. Portfolios constructed under $\mathbf{R}_{99 \%, 99 \%}$ ratio are exposed to lower downside tail risks. Additionally, it can be concluded from distributional analysis of portfolio under TSSV models that NTSSV model outperforms CTSSV model and VGCIR model in describing portfolio returns dynamics, providing more reliable consequences relative to other models.

\section{Acknowledgments}

We sincerely thank our anonymous referee. We also would like to acknowledge the financial support from the National Science Foundation of China (No. 71571038, 71671030).

\section{References}

Ait-Sahalia, Y., Jacod, J., Li, J., 2012. Testing for jumps in noisy high frequency data. J. Econ.168 (2), 207-222.

Andreas, K.A., Carol, A., 2013. Stochastic volatility jump-diffusions for European equity index dynamics. Eur. Financ. Manag. 19 (3), 470-496.

Barndorff-Nielsen, O. E., 1997. Processes of normal inverse Gaussian type. Financ. Stoch. 2 (1), 41-68.

Carr, P., Geman, H., Madan, D., Yor, M., 2002. The fine structure of asset returns: An empirical investigation. J. Bus. 75 (2), 205-332.

Carr, P., Geman, H., Madan, D., Yor, M., 2003. Stochastic volatility for Lévy processes. Math. Financ. 13 (3), 345-382.

Carr, P., Wu, L.R., 2004. Time-changed Lévy processes and option pricing, J. Financ. Econ. 71 (1), 113-141.

Choi, J., 2015. Reward-risk momentum strategies using classical tempered stable distribution. J. Bank. Financ. 58 (9), 194-213. 
Forsberg, L., Bollerslev, T., 2002. Bridging the gap between the distribution of realized (ECU) volatility and ARCH Modelling (of the Euro): The GARCH-NIG Model. J. Appl. Econ. 17 (5), 535-548.

Gajda, J., Wyłomańska, A., 2013. Tempered stable Lévy motion driven by stable subordinator. Phys. A 392, $3168-3176$

Gao, C.T., Zhou, X.H., 2016. Forecasting VaR and ES using dynamic conditional score models and skew Student distribution. Econ. Model. 53, 216-223.

Gong, X.L., Zhuang, X.T. Option pricing for stochastic volatility model with infinite activity Lévy jumps. Phys. A $455,1-10$.

Goode, J., Kim, Y.S., Fabozzi, F.J. 2015. Full versus quasi MLE for ARMA-GARCH models with infinitely divisible innovations. Appl. Econ. 47 (48), 5147-5158.

Georgiev, K., 2015. Periodic portfolio revision with transaction costs. Math. Meth. Oper. Res. 81 (3), 337-359.

Han, C.H., Liu, W.H., Chen, T.Y., 2014. VaR/CVaR estimation under stochastic volatility models. Int. J. Theo. Appl.Financ. 17 (2), 1-35.

Huang, A.Y., 2015. Value at risk estimation by threshold stochastic volatility model. Appl. Econ. 47 (45), 4884-4900.

Huang, J., Wu, L., 2004. Specification analysis of option pricing models based on time-changed Lévy processes. J. Finance, 59 (3), 1405-1440.

Liu, B., 2015. A new risk measure and its application in portfolio optimization: The SPP-CVaR approach. Econ. Model. 51, 383-390.

Kim, Y.S., Rachev, S.T., 2010a. Computing VaR and AVaR in infinitely divisible distribution. Prob. Math. Stat. 30 (2), 223-245.

Kim, Y.S., Rachev, S.T., Bianchi, M.L., Fabozzi, F.J., 2008. Financial market models with Lévy processes and time varying volatility. J. Banking. Financ. 32 (7), 1363-1378.

Klingler, S., Kim, Y. S., Svetlozar, T., 2013. Option pricing with time-changed Lévy processes. Appl. Financ. Econ. 23 (15), 1231-1238.

Kuchler, U., Tappe, S., 2013. Tempered stable distributions and processes. Stoch. Process Appl. 123 (12), 4256-4293.

Madan, D., Carr, P., Chang, E., 1998. The variance gamma process and option pricing. Eur. Financ. Rev. 2 (1), 79-105.

Menn, C., Rachev. S.T., 2006. Calibrated FFT-based density approximations for $\alpha$-stable distributions. Comput. Stat. Data Anal. 50 (8), 1891-1904.

Ouadghiri, I.E., Uctum, R., 2016. Jumps in equilibrium prices and asymmetric news in foreign exchange markets. Econ. Model. 54, 218-234.

Rachev, S.T., 2007. Momentum strategies based on reward-risk stock selection criteria. J. Bank. Financ. 31 (8), 2325-2346.

Rosinski, J., 2007. Tempering stable processes, Stoch. Proc. Appl. 117 (6), 677-707.

Ruan, X.F., 2016. Equilibrium asset pricing under the Lévy process with stochastic volatility and moment risk premiums. Econ. Model. 54, 326-338.

Sato, K., 1999. Lévy Processes and Infinitely Divisible Distributions. Cambridge University Press, Cambridge.

Scherer, M., Rachev, S.T., Kim, Y.S., Fabozzi, F.J., 2012. Approximation of skewed and leptokurtic return distributions. Appl. Financ. Econ. 22 (16), 1305-1316.

Schoutens, W., Symens, S., 2003. The pricing of exotic options by Monte-Carlo simulations in a Levy market with stochastic volatility. Int. J. Theor. Appl. Financ. 6 (8), 839-864.

Shi, Y.L., Feng, L.B., 2016. A discussion on the innovation distribution of the Markov regime-switching GARCH model. Econ. Model. 53, 278-288.

Su, Y.C., Huang, H.C., Lin, Y.J., 2011. GJR-GARCH model in value-at-risk of financial holdings. Appl. Financ. Econ. 21 (24), 1819-1829.

Stoyanov, S.V., Rachev, S.T., Fabozzi, F.J., 2013. CVaR sensitivity with respect to tail thickness. J. Bank. Financ. 37 (3), 977-988.

Todorov, V., 2011. Econometric analysis of jump-driven stochastic volatility models. J. Econometrics, 160 (1), $12-21$.

Wilhelmsson, A., 2009. Value at Risk with time varying variance, skewness and kurtosis-the NIG-ACD model. Econometric. J. 12 (1), 82-104.

Zaevski, T.S., Kim, Y.S., Fabozzi, F.J., 2014. Option pricing under stochastic volatility and tempered stable Lévy jumps. Int. Rev. Financ. Anal. 31 (C), 101-108 